\title{
Emotional and behavioural problems in children with language impairments and children with autism spectrum disorders
}

Article

Accepted Version

Charman, T., Ricketts, J., Dockrell, J. E., Lindsay, G. and Palikara, O. (2015) Emotional and behavioural problems in children with language impairments and children with autism spectrum disorders. International Journal of Language \& Communication Disorders, 50 (1). pp. 84-93. ISSN 1368-2822 doi: https://doi.org/10.1111/1460-6984.12116 Available at https://centaur.reading.ac.uk/37253/

It is advisable to refer to the publisher's version if you intend to cite from the work. See Guidance on citing.

To link to this article DOI: http://dx.doi.org/10.1111/1460-6984.12116

Publisher: Wiley

All outputs in CentAUR are protected by Intellectual Property Rights law, including copyright law. Copyright and IPR is retained by the creators or other copyright holders. Terms and conditions for use of this material are defined in the End User Agreement. 


\section{CentAUR}

Central Archive at the University of Reading

Reading's research outputs online 
Emotional and Behavioural Problems in Children with Language Impairments and Children with Autism Spectrum Disorders

Tony Charman ${ }^{1}$, Jessie Ricketts ${ }^{2}$, Julie E. Dockrell ${ }^{3}$, Geoff Lindsay ${ }^{4}$, Olympia Palikara $^{5}$

1 King's College London, Institute of Psychiatry, Department of Psychology

2 Institute of Education, University of Reading

3 Department of Psychology and Human Development, Institute of Education, University of London

4 Centre for Educational Development, Appraisal and Research (CEDAR), University of Warwick

5 School of Education, University of Roehampton

Keywords: Language impairment, Autism spectrum disorders, Emotional and behavioural problems, Mental health

Total word count $=6,336$

Tables $=3$

References $=38$ 


\begin{abstract}
Background: Although it is well-established that children with language impairment (LI) and children with autism spectrum disorders (ASD) both show elevated levels of emotional and behavioural problems, the level and types of difficulties across the two groups have not previously been directly compared.
\end{abstract}

Aims: To compare levels of emotional and behavioural problems in children with LI and children with ASD recruited from the same mainstream schools.

Methods \& Procedures: We measured teacher-reported emotional and behavioural problems using the Strengths and Difficulties Questionnaire (SDQ) in a sample of 5-to-13year old children with LI (N=62) and children with ASD $(\mathrm{N}=42)$ attending mainstream school but with identified special educational needs.

Outcomes \& Results: Both groups showed similarly elevated levels of emotional, conduct and hyperactivity problems. The only differences between the LI and ASD groups were on subscales assessing peer problems (which were higher in the ASD group) and prosocial behaviours (which were higher in the LI group). Overall, there were few associations between emotional and behavioural problems and child characteristics, reflecting the pervasive nature of these difficulties in children with LI and children with ASD, although levels of problems were higher in children with ASD with lower language ability. However, in the ASD group only, a measure of family social economic status was associated with language ability and attenuated the association between language ability and emotional and behavioural problems.

Conclusions \& Implications: Children with LI and children with ASD in mainstream school show similarly elevated levels of emotional and behavioural problems, which require 
monitoring and may benefit from intervention. Further work is required to identify the child, family and situational factors that place children with LI and children with ASD at risk of emotional and behavioural problems, and whether these differ between the two groups. This work can then guide the application of evidence-based interventions to these children.

[Word count $=299]$ 
Emotional and Behavioural Problems in Children with Language Impairments and Children with Autism Spectrum Disorders

\section{Introduction}

There has been long discussion, and some disagreement, within the field about whether there is overlap (or not) in the aetiology, behavioural phenomenology and language profiles of children with language impairments (LI) and children with autism spectrum disorders (ASD) (see Bishop, 2010; Tomblin, 2011; Ellis Weismer, 2013; Williams, Botting, $\&$ Boucher, 2008; for reviews). However, one area that has received less attention is the extent to which co-occurring emotional and behavioural difficulties are seen in both disorders. Previous studies have indicated high levels of emotional and behavioural problems in independent samples of children with LI and children with ASD, although rarely have these been directly compared in samples ascertained in a similar fashion. Cross-disorder comparisons are important in order to identify whether the factors that are associated with emotional and behavioural problems are similar or different in children with LI and children with ASD. This may then, in turn, inform whether similar, or different, preventative or intervention strategies may be required in order to ameliorate the effects of these difficulties.

In samples of LI children, prevalence rates of emotional and behavioural problems have been as high as 35\%-50\% (Lindsay, Dockrell, \& Strand, 2007; van Daal, Verhoeven, \& van Balkom, 2007; for a review see Yew \& O’Kearney, 2013). Both elevated rates of externalising difficulties (hyperactivity, conduct problems, oppositional behaviour) and internalising difficulties (anxiety, depression) have been reported (Conti-Ramsden \& Botting, 2008; Redmond \& Rice, 2002; Snowling, Bishop, Stothard, Chipchase, \& Kaplan, 2006; Tomblin, Zhang, Buckwalter, \& Catts, 2000). Despite variation in the definitions and measures of emotional and behavioural problems that have been used, with many US studies 
using the Child Behaviour Checklist (CBCL; Achenbach, 2009) and many European studies the Strengths and Difficulties Questionnaire (SDQ; Goodman, 1997), elevated rates of both externalising and internalising difficulties have been found consistently. Two recent studies have tracked the longitudinal trajectory of emotional and behavioural difficulties using the SDQ in samples of children and young people with LI from middle childhood through to adolescence (Lindsay \& Dockrell, 2012; St. Clair, Pickles, Durkin, \& Conti-Ramsden, 2011). Both studies found that rates remained generally elevated compared to population norms, although the trajectory differed across the different SDQ subscales and across the two studies. In both studies, levels of hyperactivity and emotional problems decreased and levels of peer problems increased. However, conduct problems decreased over time in the St. Clair et al (2011) study but increased in the Lindsay and Dockrell (2012) study.

In samples of children with ASD, some studies have used the SDQ to index emotional and behavioural problems (e.g. Totsika, Hastings, Emerson, Lancaster, \& Berridge, 2011), whilst other studies have used diagnostic instruments, reporting rates of mental health or psychiatric disorders (e.g. Simonoff et al., 2008). Prevalence rates for emotional and behaviour problems (Emotional symptoms on the SDQ; anxiety disorders, conduct and oppositional defiant disorder) have been as high as 50\%-75\% in several studies (Leyfer et al., 2006; Simonoff et al., 2008; Totsika et al., 2011) with the most common being ADHD, anxiety and oppositional behaviour. A recent study has shown relative stability of rates of clinical-level disorders but a significant reduction in mean SDQ subscale scores in a population sample followed up from 12 to 16 years of age (Simonoff et al., 2013).

It is well established within the general child psychopathology field that child (e.g. IQ) and family (e.g. social economic status; parental mental health) factors are associated with rates of emotional and behavioural problems (Ford, Goodman \& Meltzer, 2003). 
However, there have been mixed findings in terms of child associates of emotional and behavioural problems in children with LI. In the Lindsay and Dockrell (2012) LI sample, language ability and academic achievement (reading, spelling) were not strong concurrent or longitudinal predictors of levels of emotional and behavioural problems. By contrast, in the St. Clair et al (2011) study, reading skills and expressive language were associated with later levels of emotional and behavioural problems as measured by the SDQ, such that children with poorer reading and expressive language were more likely to exhibit emotional and behavioural problems. In the Simonoff et al. (2008) ASD study neither child nor family factors were associated with rates of emotional and behavioural problems.

From the studies conducted to date, it is difficult to ascertain the extent to which the levels and profile of emotional and behavioural problems are similar or different in children with LI and ASD. This is due to a number of methodological factors that vary across the studies conducted. These range from potential biases due to sample ascertainment, whereby clinically-ascertained samples of children with LI and children with ASD may be more likely to have emotional and behavioural problems than school-ascertained or population samples, to the use of different measures used across studies, as well as sample differences in age and IQ. No studies to date have directly compared the level and profiles of emotional and behavioural problems in samples of children with LI and ASD recruited in parallel from the same schools. The present study aimed to compare and contrast the level and profile of emotional and behavioural problems in a mainstream school-ascertained sample of children with LI and children with ASD.

Our research questions were:

- What levels of emotional and behavioural problems do children with LI and children with ASD in mainstream school exhibit? 
- Does the profile of emotional and behavioural problems differ between the two groups?

- Are levels of emotional and behavioural difficulties related to age, nonverbal abilities, language abilities, and level of autistic symptomatology, and are these associations similar or different in the two groups?

\section{Method}

Participants for this study comprised 104 school-aged participants with LI ( $n=62 ; 46$ male, 16 female) or $\operatorname{ASD}(n=42 ; 36$ male, 6 female) with valid SDQ data, drawn from the total sample of 157 (66.2\%) who were recruited as part of a large UK study the Better Communication Research Programme (Dockrell, Lindsay, Law \& Roulstone, in press). ANOVAs were run to test if the subsamples of participants with available SDQ data systematically differed from those without. No differences were found between participants with and without SDQ data on age (Time 2), NVIQ (BAS-II Matrices), language (BPVS, CELF-CFD, TROG) and autism severity score (SRS) (all $p>.18$ ).

Participants were recruited from 74 mainstream primary and secondary schools across five Local Authorities in the South East of England. Following agreement from the schools, pupils were identified who were aged $6,8,10$, and 12 years and had speech, language and communication needs (SLCN) or ASD as their primary special educational needs (SEN), according to their school. All pupils spoke English as a first language and had no history of hearing impairment or uncorrected eyesight. In the UK, the Pupil Level School Census requires schools to identify and notify the Department for Education (DfE) of pupils with SEN.

Pupils with a School Census primary classification of ASD will have received a medical diagnosis of an autism spectrum disorder using ICD-10 (WHO, 1993) or DSM-IV 
(APA, 2000) criteria via community child health or specialist paediatric or child psychiatric service. For SLCN, identification would include a range of clinically- and school-ascertained needs and diagnoses relating to speech, language and/or communication difficulties. From students with SLCN, we were interested in recruiting students with oral language impairments. Given the lack of an agreed measure for identifying language impairment (Bishop \& McDonald, 2009) we conducted a screening phase. Students were identified as having LI if they obtained a standardized score that was below the average range i.e. greater than one standard deviation below the mean $(<1 \mathrm{SD})$ on either the Recalling Sentences or Word Classes subtest from the CELF-4 UK (Semel, Wiig, \& Secord, 2006), at which point the remaining relevant subscales of the CELF-4 UK were administered.

At the screening phase teachers were asked to complete the Social Responsiveness Scale (SRS: Constantino \& Gruber, 2005) as a continuous measure of autistic symptomatology. The SRS was completed for 98 pupils across the LI $(n=58 ; 94 \%)$ and ASD $(n=40 ; 95 \%)$ groups. For this measure, a high score indicates greater impairment. A majority of participants with ASD obtained scores that were more than one standard deviation above the test mean $(n=31,78 \%)$, although a minority did so in the LI group ( $n=$ $21,36 \%)$.

Ethical agreement was provided for the study by the University of Warwick Humanities and Social Science Research Ethics Committee, which adheres to the British Psychological Society guidelines.

\section{Materials and procedure}

The tasks reported here form part of a larger assessment battery and were administered at three time points (screening phase, Time 1, Time 2). Between screening and 
Time 1 there was a mean time difference of 3.9 months $(S D=3.0)$ and between Time 1 and Time 2 there was a mean time difference of 7.4 months $(S D=3.3)$.

Nonverbal reasoning ability (NVIQ). The Matrices subtest from the British Ability Scales-II (BAS-II; Elliot, Smith, \& McCulloch, 1997) was administered at the screening phase as a measure of NVIQ. In this task, participants are presented with an incomplete pattern and are required to select the picture that will complete the pattern. It produces a $T$ score $(M=50, S D=10)$.

Language ability. The Recalling sentences and Word classes subtests from the clinical Evaluation of Language Fundamentals (CELF-4 UK: Semel et al., 2006) were also administered during the screening phase and used to confirm oral language impairments in LI group. In the Recalling sentences subtest participants repeat orally presented sentences. In the Word classes subtest, upon hearing three or four words read aloud by the examiner, pupils are asked to select the two words that go together. Pupils are then asked to explain the connections in meaning between the two words they selected. Each subtest produces a scaled score $(M=10, S D=3)$.

At Time 1 pupils completed the following three language measures:

The third edition of the British Picture Vocabulary Scale (BPVS-3: Dunn, Dunn, \& NFER, 2009). In this receptive vocabulary task, participants hear a word and are required to indicate what it means by selecting a picture from four alternatives. It produces a standardised score $(M=100, S D=15)$

The Test for Reception of Grammar (TROG-E: Bishop, 2005) provided a measure of receptive grammar. In the TROG, individuals hear a series of sentences that increase in grammatical complexity and indicate comprehension by selecting a picture from four alternatives. It produces a standardised score $(M=100, S D=15)$ 
Participants completed the Concepts and following directions subtest from the (CELF-4 UK: Semel et al., 2006). The subtest requires the participant to identify objects in response to oral directions and produces a scaled score $(M=10, S D=3)$.

In order to reduce the number of variables, a composite mean z-score of performance on the BPVS, CELF-CFD and TROG was calculated (all pairwise $r>.53, p<.001$ ). These measures did not form part of the screening phase to identify children with LI.

Autism symptomatology. The SRS (Constantino \& Gruber, 2005) was completed by teachers during the screening phase. Respondents are presented with a series of statements relating to autism symptomatology and indicate the frequency of their occurrence. The SRS generates a total $T$-score $(M=50, S D=10)$ with higher scores reflecting higher level of autistic behaviours.

Emotional and behavioural problems. Teachers completed the Strengths and Difficulties Questionnaire (SDQ: Goodman, 1997) at Time 2. The SDQ comprises 5 subscales: Hyperactivity, Conduct problems, Emotional symptoms, Peer problems and Prosocial behaviour. Screening cut-offs (www.sdqinfo.com) are available to identify children with 'abnormal' levels of difficulties - the highest $10 \%$ ile from over 8,000 teacher ratings (Meltzer, Gatward, Goodman, and Ford, 2000). Each item is scored 0-2, with subscale scores across 5 items ranging from 0 - 10; higher scores indicating higher levels of psychopathology (with the exception of the Prosocial behaviour subscale where lower scores indicate higher levels of psychopathology). The Hyperactivity, Emotional symptoms, Conduct problems and Peer relationship subscales comprise the Total Difficulties scale (range 0 - 40).

Family background characteristics. Two indices of socio-economic status (SES) were provided by the School Census data; whether or not the pupil was currently eligible for free school meals (FSM) and their Income Deprivation Affecting Children Index (IDACI) score. 
IDACI scores were derived from the pupils' postcodes (Department for Education, 2013). The proportion of the current sample that was eligible for FSM (25\%) was slightly higher than the national UK average (18\%).

\section{Results}

Pupils in the ASD group were significantly older than pupils with LI at the screening phase, $F(1,102)=6.92, p=.01$, Time $1, F(1,102)=7.28, p=.008$ and Time $2, F(1,102)=6.78, p=$ .01. The groups did not differ in terms of NVIQ. The LI group had significantly lower scores than the ASD group on the language measures, BPVS: $F(1,101)=16.02, p<.001$; CELF-CFD: $F(1,97)=18.37, p<.001$; TROG: $F(1,100)=5.60, p=.02$. The ASD group had significantly higher scores, indicating higher levels of autistic behaviours, on the SRS, $F(1,96)=21.96, p<.001$. For both the LI and ASD groups scores on all three language measures were lower (using comparable z-scores on all measures) than NVIQ (paired $t$-tests; minimum $t$-score value 4.72, all $p<.001$ ).

\section{----- Table 1 about here -----}

SDQ scores and the proportion scoring above population $90^{\text {th }}$ percentile ('abnormal range') for the LI and ASD groups are shown in Table 2. Both groups showed elevated scores compared to population norms, with between $18 \%$ and $37 \%$ of the LI group and $21 \%$ and $67 \%$ of the ASD group falling above the clinical $90^{\text {th }}$ percentile cut-off, depending on the subscale. Nonparametric sign tests showed that for the LI group the proportion scoring above the $90^{\text {th }}$ percentile was higher for the Emotional symptoms subscale than the Prosocial subscale $(p<.05)$. For the ASD group, the proportion scoring above the $90^{\text {th }}$ percentile was higher for the Peer problems subscale than on all four other scales (Emotional symptoms $p<$ .05 , all three other subscales $p<.001)$. An omnibus MANOVA showed that the groups differed across the 6 subscales and total score, $F(1,99)=4.67, p<.001$. Follow-up ANOVAs 
showed that the groups did not differ on the Total Difficulties score or on the Emotional symptoms $F(1,102)=0.00, p=.99$, Conduct problems, $F(1,100)=0.06, p=.80$, and Hyperactivity subscales, $F(1,102)=0.09, p=.77$. The ASD group scored higher than the LI group on the Peer problems subscale, $F(1,101)=15.21, p<.001$, and lower on the Prosocial subscale, $F(1,100)=5.42, p=.02$.

\section{----- Table 2 about here -----}

In order to examine whether child characteristics were associated with levels of emotional and behavioural problems, correlations between age, NVIQ, language ability, autism symptom severity and scores on the SDQ are shown separately for each group in Table 3. Given the wide range of age in the sample, age-partialled correlations between SDQ scores and NVIQ, language ability and autism symptom severity are also shown. Given the large number of comparisons only associations significant at the $p<.01$ level are highlighted. In the LI group, only two associations were significant; teacher-reported Peer problems were positively associated with age $(r=.36, p=.005)$ and Emotional symptoms were positively associated with autism symptom severity as measured by the SRS $(r=.40, p=.002)$ and remained so when the effect of age was partialled out $(r=.42, p=.001)$. In the ASD group, two subscales (Emotional symptoms, $r=-.42, p=.009$; Hyperactivity, $r=-.45, p=.004$ ) and the Total Difficulties score $(r=-.55, p<.001)$ were negatively associated with the language composite, indicating increased emotional and behavioural problems in those children with poorer language abilities. The negative association between Total Difficulties score and language abilities remained after age was partialled out $(r=-.49, p=.002)$ but those for Emotional symptoms and Hyperactivity fell below our adjusted significance level (both $r=-$ $.37, p=.02)$. 
We also examined whether levels of emotional and behavioural problems were associated with the background family characteristics that were available on the sample: children eligible for free school meals (FSM) and IDACI score. The former was tested within each group by a series of independent samples t-tests comparing scores for those who were, and who were not, eligible for FSM. The latter was tested by Pearson's correlations. None of the associations reached our conservative significance level of $p<.01$ but for the LI group Peer problems were marginally higher in children eligible for $\operatorname{FSM}(p=.04)$ and in the ASD group Conduct problems were marginally associated with increased deprivation as measured by the IDACI score $(p=.03)$. We then explored whether either eligibility for FSM or IDACI scores was associated with NVIQ, language and SRS score, again analysing each group separately. In the ASD group only IDACI score was moderately associated with the language composite $(\mathrm{r}=.-47, p<.001)$ and when this was partialled out (in addition to age) in the correlations between child characteristics and SDQ scores, the negative association between language ability and levels of emotional and behavioural problems was attenuated but still marginally significant for the SDQ Total problems score $(r=-.41, p<.05)^{1}$.

\section{Discussion}

Levels of emotional and behavioural problems in the two groups

We measured teacher-reported emotional and behavioural problems in children with LI and children with ASD attending mainstream schools in the UK. Both groups showed significantly elevated levels of emotional and behavioural problems, compared to population norms, as measured by the SDQ. The only differences between the LI and ASD groups were on subscales assessing social difficulties (higher levels of peer problems, lower levels of prosocial behaviour), which were higher in the ASD group, reflecting the core social

\footnotetext{
${ }^{1}$ Details of the analysis available from the first author
} 
communication impairments that characterise this group. However, on the Emotional symptoms, Conduct problems and Hyperactivity subscales of the SDQ, between $20 \%$ and $40 \%$ of the children in both groups scored above the population $90^{\text {th }}$ percentile range (and their mean scores did not differ from one another), indicating possible clinical levels of difficulty (Goodman, 1997). In terms of peer problems one third of the LI group and two thirds of the ASD group scored above this threshold. Looking within each group at the proportion scoring above the $90^{\text {th }}$ percentile, the only notable pattern was for more children with ASD to score in the elevated range on the Peer problems subscale, in line with the difficulties in social interaction and peer-relating required for a diagnosis of ASD. In summary, the pattern for both the LI and ASD groups was to show elevated scores on the SDQ subscales measuring broad emotional and behaviour psychopathology: emotional problems, hyperactivity and conduct problems.

The findings confirm previous research that has found elevated levels of emotional and behavioural problems in separate samples of children with LI (Conti-Ramsden \& Botting, 2008; Lindsay et al., 2007; Lindsay \& Dockrell, 2012; St. Clair et al., 2011; van Daal et al., 2007) and children with ASD (Leyfer et al., 2006; Simonoff et al., 2008; Totsika et al., 2011). For both groups, the types of emotional and behavioural problems that were elevated are similar to findings reported in previous studies, including conduct problems, hyperactivity and emotional problems. What this study adds is the direct comparison of the levels of emotional and behavioural problems in children with LI and children with ASD recruited in parallel from the same mainstream schools and assessed on the same measure. Both groups showed elevated levels of emotional problems, hyperactivity and conduct problems, similar in level to previous cohorts of children with SLI recruited from specialist language units (Wadman et al., 2011) and mainstream schools (Lindsay \& Dockrell, 2000) 
but lower than in a (slightly older) population sample of children with ASD (Simonoff et al., 2013).

Associations between levels of emotional and behavioural problems and child characteristics

In addition, we systematically examined associations between levels of emotional and behavioural problems in both groups and child characteristics known to affect behaviour (NVIQ, language ability, autism severity). In the LI group, higher levels of teacher-rated emotional symptoms were associated with higher levels of behaviours characteristic of ASD. In the ASD group, levels of emotional and behavioural problems were negatively associated with language abilities, indicating that in this group better language abilities might protect against emotional and behavioural problems. By definition, the children with LI all had lower language ability, and this restricted variability in language may explain the lack of such an effect in this group (see Table 1). Our findings do not allow us to identify what these protective mechanisms might be but for at least some verbally able children with ASD, it might be that better language and communication skills buffer the effects of poor intuitive social insight and understanding, or provide compensatory routes to adaptive social interactions and well-being. Understanding protective mechanisms will be important for guiding support and interventions.

Overall, there were few strong associates of levels of emotional and behavioural difficulties even though there was considerable variation in child characteristics such as NVIQ and language abilities across the sample, reflecting the pervasive nature of these difficulties across the two groups. Language comprehension difficulties have been associated with other areas of development in children with LI, such as quality of friendships (Durkin \& Conti-Ramsden, 2007). Some previous studies have also found that levels of emotional and behavioural problems are negatively associated with expressive language ability (and reading 
ability) in children with LI (St. Clair et al., 2011), although other studies have failed to find associations with receptive language abilities, consistent with the present findings (Lindsay \& Dockrell, 2012). Previous studies with children with ASD have failed to find associations with child characteristics such as IQ (Simonoff et al., 2008), which are known to be strongly associated with psychopathology in the general population (Ford, Goodman, \& Meltzer, 2003).

Our finding that child characteristics were only weakly predictive of levels of emotional and behavioural problems indicates that other factors, such as family functioning, school environment and peer interactions likely influence emotional functioning and behaviour in individuals with these conditions. The current study had only limited data on possible social and demographic factors and found only marginal associations between eligibility for free school meals and neighbourhood deprivation and emotional and behavioural problems. Further, in the ASD group neighbourhood deprivation was associated with language ability and attenuated the association with emotional and behavioural problems. It is well known that social factors can affect both developmental skills and mental health (Emerson \& Einfeld, 2010). There are a wide range of family characteristics that might act as risk or protective factors for emotional and behavioural problems (e.g. parenting behaviour, parental mental health). These issues require further study to understand the factors that make children with LI and children with ASD vulnerable to emotional and behavioural problems and in particular how child and family/background factors might interact over time to increase vulnerability (see Midouhas et al., 2013; for a recent exception in young children with ASD).

\section{Clinical implications}


Our findings have clear clinical implications. Notably, those caring for and working with children with LI and children with ASD need to be aware of their generalised vulnerability for emotional and behavioural problems. When formal psychiatric disorders have been systematically assessed they have been shown to be common in other samples of children with LI (Beitchman et al., 2001) and ASD (Simonoff et al., 2008) and those working with children with LI and ASD need to be aware of their vulnerability to such outcomes.

However, our understanding both of the factors that might place children with LI and children with ASD at risk (or protect them from) emotional and behavioural problems is still partial. Although there are well-established psychological interventions for emotional and behavioural problems that many children and young people experience (James et al., 2013; Sonuga-Barke et al., 2013), it cannot be assumed that they will also be effective when cooccurring in children with development disabilities such as LI and ASD. Indeed, the recent NICE guidelines on the management of children and young people with ASD pointed to trials that have modified aspects of the content and delivery of cognitive behavioural therapy for anxiety in children with ASD (NICE, 2013).

The pattern of associations between childhood factors such as language ability and IQ and emotional and behavioural problems in our sample was not strong (with the exception of poor language abilities being associated with higher levels of problem in the ASD but not the LI group), and contrary to that seen in the general child psychopathology field (Ford et al., 2003). Another assumption that requires testing is whether the nature (and experience) of internalising and externalising difficulties is similar or different in children with LI and ASD as it is in those without (Ellis Weismer, 2013; Simonoff et al., 2013). For example, both developmental and cognitive mechanisms help us understand, in part, why evidence-based interventions for these conditions are effective. Further research examining these factors in 
children with co-occurring LI and ASD and emotional and behavioural problems will help reveal the potential for these interventions to be applicable to this population, or suggest how they might need modifying.

\section{Limitations}

The present study has several strengths, most notably the likely generalisability of the findings given the way that the sample was ascertained from mainstream schools. Nonetheless, several limitations are worthy of mention. Identification of the sample and classification into the ASD group relied on the UK School Census categorisation of ASD. Whilst in almost all cases this would only follow a community medical diagnosis of ASD no independent clinical assessment was conducted to confirm these diagnoses; similarly, we did not conduct direct assessments to rule out ASD in the LI group. However, on the SRS screening measure the ASD group scored almost 2 standard deviations above the population norms and within the range reported in well-characterised clinical samples (Constantino et al., 2007; Schanding, Nowell, \& Goin-Kochel, 2012). When analyses were re-run only including those LI children who fell below a $T$-score of 60 on the SRS and those ASD children who scored above 60 the pattern of results remained the same. It is worth also noting that our reliance on teacher ratings on the SRS only, and in the absence of parental report, is a limitation of the current study, in part because children's behaviour may be different in different settings, but also because the same rater completed both measures of psychopathology (SDQ and SRS), thus scores were vulnerable to rater 'halo effects'.

Another limitation is the reliance on the SDQ, which is a screening measure to characterise emotional and behavioural problems, with no independent clinical psychiatric assessment. Whilst the SDQ has been shown to have good psychometric properties against psychiatric diagnoses (Goodman, 2001), its validity as a screening measure in children with 
LI and ASD requires further confirmation (Simonoff et al., 2013). In terms of the association between language abilities and emotional and behavioural problems, we only examined the association on a receptive language composite measure and future studies are needed in order to examine whether receptive and expressive language abilities might have separate or different effects on emotional and behavioural problems. Further, we did not systematically measure parental, family, peer interaction and school characteristics that are known to be associated with child wellbeing and adjustment. Finally, both groups had an insufficient number of girls for gender differences to be statistically examined and future samples with large number of girls are needed to examine whether different factors might be associated with emotional and behavioural problems in boys and girls.

\section{Conclusions}

Children with LI and children with ASD in mainstream school showed elevated levels of emotional and behavioural problems, which require monitoring, and may benefit from intervention. Further research is required to understand the risk and protective factors that lead to the different profiles of social-emotional functioning and behaviour in children with LI and children with ASD in mainstream schools.

\section{What is known on this topic:}

- Several studies has found elevated levels of emotional and behavioural problems in children with LI and children with ASD

- No studies to date have directly compared levels of emotional and behavioural problems in children with LI and children with ASD recruited via mainstream schools.

\section{What this paper adds:}


- Children with LI and children with ASD in mainstream school showed similar and highly elevated levels of emotional, conduct and hyperactivity problems.

- Child characteristics such as age, nonverbal abilities and language abilities were only weakly associated with levels of emotional and behavioural problems, although lower language abilities were associated with elevated levels of emotional problems in children with ASD.

- Those caring for and working with children with LI and children with ASD need to be aware of their generalised vulnerability for emotional and behavioural problems, these require monitoring, and may benefit from intervention.

- Further work to identify risk and protective factors, and developmental and cognitive mechanisms, associated with emotional and behavioural problems in children with LI and children with ASD is required in order to inform how interventions might need to be modified for these groups. 
Acknowledgements: We would like to acknowledge the support of all the students, their families and schools. Without their help the project could not have been completed. We are particularly grateful to Nita Patel, Shona Kelly and Melissa Hollinworth for assistance with data collection and project planning. This research was funded by the UK Department for Education as part of the Better Communication Research Programme:

http://www.education.gov.uk/researchandstatistics/research/better.

Conflict of interest declaration: The authors have no conflicts of interest to declare. 


\section{References}

ACHENBACH, T. M. (2009). The Achenbach System of Empirically Based Assessemnt (ASEBA): Development, Findings, Theory, and Applications. Burlington, VT: University of Vermont Research Center for Children, Youth and Families.

AMERICAN PSYCHIATRIC ASSOCIATION (2000). Diagnostic and Statistical Manual of Mental Disorders. (4th, Text Revision (DSM-IV-TR) ed.) Washington, DC: American Psychiatric Association.

BEITCHMAN, J. H., WILSON, B., JOHNSON, C. J., ATKINSON, L., YOUNG, A., ADLAF, E.,...DOUGLAS, L. Fourteen-year follow-up of speech/language impaired and control children: Psychiatric outcomes. Journal of the American Academy of Child and Adolescent Psychiatry, 40, 75-82.

BISHOP, D. V. M. (2005). Test of Reception for Grammar - Electronic (TROG-E). London: Harcourt Assessment.

BISHOP, D. V. (2010). Overlaps between autism and language impairment: phenomimicry or shared etiology? Behavior Genetics, 40, 618-629.

BISHOP, D. V., \& McDONALD D. (2009). Identifying language impairment in children: combining language test scores with parental report. International Journal of Language and Communication Disorders, 44(5), 600-615.

CONSTANTINO, J., \& GRUBER, C. (2005). The Social Responsiveness Scale. Los Angeles: Western Psychological Services. 
CONSTANTINO, J. N., LAVESSER, P. D., ZHANG, Y., ABBACCHI, A. M., GRAY, T., \& TODD, R. D. (2007). Rapid quantitative assessment of autistic social impairment by classroom teachers. Journal of the American Academy of Child and Adolescent Psychiatry, $46,1668-1676$.

CONTI-RAMSDEN, G. \& BOTTING, N. (2008). Emotional health in adolescents with and without a history of specific language impairment (SLI). Journal of Child Psychology and Psychiatry, 49, 516-525.

DEPARTMENT FOR EDUCATION. (2013). Income Deprivation Affecting Children Index (IDACI). http://www.education.gov.uk/cgi-bin/inyourarea/idaci.pl (accessed 31 October 2013).

DOCKRELL, J., LINDSAY, G., LAW, J., \& ROULSTONE, S. (in press). Supporting children with speech language and communication needs: an overview of the results of the Better Communication Research Programme, International Journal of Language and Communication Disorders.

DUNN, L. M., DUNN, D. M., \& NFER. (2009). British Picture Vocabulary Scale - 3 (3rd ed.). London: GL Assessment Ltd.

DURKIN, K., \& CONTI-RAMSDEN, G. M. (2007). Language, social behaivour, and the quality of friendships in adlescents with and without a history of specific language impairment. Child Development, 78, 1441-1457.

ELLIOTT, C. D., SMITH, P., \& MCCULLOCH, K. (1997). British Ability Scales II. London: GL Assessment. 
ELLIS WEISMER, S. (2013).Developmental language disorders: Challenges and implications of cross-group comparisons. Folia Phoniatrica et Logopaedica, 65, 68-77.

EMERSON, E., \& EINFELD, S. (2010). Emotional and behavioural difficulties in young children with and without developmental delay: a bi-national perspective. Journal of Child Psychology and Psychiatry, 51, (5), 583-593.

FORD, T., GOODMAN, R., \& MELTZER, H. (2003). The British Child and Adolescent Mental Health Survey 1999: the prevalence of DSM-IV disorders. Journal of the American Academy of Child \& Adolescent Psychiatry, 42(10), 1203-11

GOODMAN, R. (1997). The strengths and difficulties questionnaire: A research note. Journal of Child Psychology and Psychiatry, 38(5), 581-586.

GOODMAN, R. (2001). Psychometric properties of the strengths and difficulties questionnaire. Journal of the American Academy of Child and Adolescent Psychiatry, 40(11), 1337- 1345 .

JAMES, A. C., JAMES, G., COWDREY, F. A., SOLER, A., \& CHOKE A. (2013). Cognitive behavioural therapy for anxiety disorders in children and adolescents.

Cochrane Database of Systematic Reviews. 2013, Issue 6.

LEYFER, O. T., FOLSTEIN, S. E., BACALMAN, S., DAVIS, N. O., DINH, E., MORGAN, J., ... LAINHART J. E. (2006). Comorbid psychiatric disorders in children with autism: Interview development and rates of disorders. Journal of Autism and Developmental Disorders, 36, 849-861. 
LINDSAY, J., \& Dockrell, J. E. (2000). The behaviour and self-esteem of children with specific speech and language difficulties. British Journal of Educational Psychology, 70, 583-601.

LINDSAY, G., DOCKRELL, J. E., \& STRAND, S. (2007). Longitudinal patterns of behaviour problems in children with specific speech and language difficulties: child and contextual factors. British Journal of Educational Psychology, 77, 811-828.

LINDSAY, G., \& DOCKRELL, J. E. (2012). Longitudinal patterns of behavioral, emotional, and social difficulties and self-concepts in adolescents with a history of specific language impairment. Language Speech and Hearing Services in Schools, 43, 445-60.

MIDOUHAS, E., YOGARATNAM, A., FLOURI, E., \& CHARMAN, T. (2013) Trajectories of psychopathology in young children with autism spectrum disorder: The role of family poverty and parenting. Journal of the American Academy of Child and Adolescent Psychiatry, $52,1057-1065$.

NATIONAL INSTITUTE FOR HEALTH AND CARE EXCELLENCE (NICE). (2013). Autism: Management of Autism in Children and Young People (CG170). London: NICE. REDMOND, S. M. \& RICE, M. L. (2002). Stability of behavioral ratings of children with SLI. Journal of Speech Language and Hearing Research, 45, 190-201.

SCHANDING, G. T., NOWELL, K. P., \& GOIN-KOCHEL, R. P. (2012). Utility of the Social Communication Questionnaire-Current and Social Responsiveness Scale as teacherreport screening tools for autism spectrum disorders. Journal of Autism and Developmental Disorders, 42(8), 1705-1716. 
SEMEL, E., WIIG, E., \& SECORD, W. (2006). Clinical Evaluation of Language

Fundamentals - Fourth UK Edition (CELF-4 UK). London: Pearson Assessment.

SIMONOFF, E., PICKLES, A., CHARMAN, T., CHANDLER, S., LOUCAS, T., \& BAIRD, G. (2008). Psychiatric disorders in children with autism spectrum disorders: Prevalence,

comorbidity, and associated factors in a population-derived sample. Journal of the American Academy of Child and Adolescent Psychiatry, 47(8), 921-929.

SIMONOFF, E., JONES, C. R. G., BAIRD, G., PICKLES, A., HAPPÉ, F., \& CHARMAN, T. (2013). The persistence and stability of psychiatric problems in adolescents with autism spectrum disorders. Journal of Child Psychology and Psychiatry, 54 (2), 186-194.

SNOWLING, M. J., BISHOP, D. V. M., STOTHARD, S. E., CHIPCHASES B., \& KAPLAN, C. (2006). Psychosocial outcomes at 15 years of children with a preschool history of speech-language impairment. Journal of Child Psychology and Psychiatry, 47(8), 759765.

SONUGA-BARKE, E. J., BRANDEIS, D., CORTESE, S., DALEY, D., FERRIN, M., HOLTMANN, M.... European ADHD Guidelines Group. (2013). Nonpharmacological interventions for ADHD: systematic review and meta-analyses of randomized controlled trials of dietary and psychological treatments. American Journal of Psychiatry, 170(3), 275289.

ST CLAIR, M. C., PICKLES, A., DURKIN, K., \& CONTI-RAMSDEN, G. (2011). A longitudinal study of behavioral, emotional and social difficulties in individuals with a history of specific language impairment (SLI). Journal of Communication Disorders, 44(2), 186-199. 
TOMBLIN, J. B., ZHANG, X. Y., BUCKWALTER, P., \& CATTS, H. (2000). The association of reading disability, behavioral disorders, and language impairment among second-grade children. Journal of Child Psychology and Psychiatry, 41, 473-482.

TOMBLIN, B. (2011). Co-morbidity of autism and SLI: kinds, kin and complexity. International Journal of Language and Communiccation Disorders, 46, 127-137

TOTSIKA, V., HASTINGS, R. P., EMERSON, E., LANCASTER, G. A., \& BERRIDGE, D. M. (2011). A population-based investigation of behavioural and emotional problems and maternal mental health: associations with autism spectrum disorder and intellectual disability.

Journal of Child Psychology and Psychiatry, 52, 91-99.

VAN DAAL, J., VERHOEVEN, L., VAN BALKOM, H. (2004). Subtypes of severe speech and language impairments: Psychometric evidence from 4-year-old children in the Netherlands. Journal of Speech Language and Hearing Research, 47, 1411-1423.

WADMAN, R., BOTTING, N., DURKIN, K., \& CONTI-RAMSDEN, G. (2011). Changes in emotional health symptoms in adolescents with specific language impairment. International Journal of Language and Communication Disorders, 46, 641-656.

WILLIAMS, D., BOTTING, N., \& BOUCHER, J. (2008). Language in autism and specific language impairment: where are the links? Psychological Bulletin, 13, 944-963.

WORLD HEALTH ORGANIZATION (1993). Mental disorders: a glossary and guide to their classification in accordance with the $10^{\text {th }}$ revision of the international classification of diseases-research diagnostic criteria (ICD-10). Geneva: WHO. 
YEW, S. G., \& O'KEARNEY, R. (2013). Emotional and behavioural outcomes later in childhood and adolescence for children with specific language impairments: meta-analyses of controlled prospective studies. Journal of Child Psychology and Psychiatry, 54, 516-524. 
Table 1. Age, NVIQ, language scores and autism severity scores for LI and ASD groups

\begin{tabular}{|c|c|c|c|}
\hline & LI & ASD & Effect size \\
\hline & Mean (SD) & Mean (SD) & Cohen's d \\
\hline & $\mathrm{N}=62$ & $\mathrm{~N}=42$ & \\
\hline Age in months (screening) & $104.95(29.62)$ & $119.40(24.00)^{*}$ & .51 \\
\hline Age in months (Time 1) & $108.73(29.51)$ & $123.40(23.42)^{* *}$ & .52 \\
\hline Age in months (Time 2) & $116.21(29.29)$ & $130.36(23.72) *$ & .51 \\
\hline BAS-II Matrices T-score & $45.57(11.32)$ & $49.236(12.71)$ & .32 \\
\hline BPVS standard score & $76.06(8.13)$ & $85.98(16.77)^{* * *}$ & .75 \\
\hline CELF-CFD scaled score & $3.95(2.59)$ & $6.64(3.65)^{* * *}$ & .81 \\
\hline TROG standard score & $75.03(13.83)$ & $83.24(21.123)^{*}$ & .47 \\
\hline SRS Total T-score & $57.17(9.99)$ & $67.98(12.80)^{* * *}$ & .87 \\
\hline
\end{tabular}

$* \mathrm{p}<0.05 ; * * \mathrm{p}<0.01 ; * * * \mathrm{p}<0.001$ 
Table 2. SDQ Total and Subscale scores for LI and ASD groups and percentage above $90^{\text {th }}$ percentile

\begin{tabular}{|c|c|c|c|c|c|}
\hline & LI & & ASD & & \\
\hline & Mean & (SD) & Mean (SD) & $\%$ & $E S^{\mathrm{a}}$ \\
\hline & $\mathrm{N}=62$ & & $\mathrm{~N}=42$ & & \\
\hline Total score & $12.77(7.41)$ & $31.67 \%$ & $14.44(7.10)$ & $41.46 \%$ & 0.23 \\
\hline Emotional symptoms & $3.29(2.77)$ & $37.10 \%$ & $3.29(2.74)$ & $40.48 \%$ & 0.00 \\
\hline Conduct problems & $1.95(2.58)$ & $24.59 \%$ & $1.83(2.14)$ & $21.95 \%$ & 0.05 \\
\hline Hyperactivity & $5.10(2.99)$ & $25.81 \%$ & $5.26(2.43)$ & $21.43 \%$ & 0.06 \\
\hline Peer problems & $2.43(2.35)$ & $34.43 \%$ & $4.31(2.49)^{* * *}$ & $* 66.67 \%$ & 0.73 \\
\hline Prosocial & $6.43(2.69)$ & $18.03 \%$ & $5.20(2.50)^{*}$ & $21.95 \%$ & 0.46 \\
\hline
\end{tabular}


Table 3.

Correlations (age partialled in parentheses) between SDQ scores and age (Time 2), NVIQ, language and SRS in the LI and ASD subgroups

\begin{tabular}{|c|c|c|c|c|}
\hline & Age & NVIQ & Language & SRS \\
\hline \multicolumn{5}{|c|}{ LI group $(N=62)$} \\
\hline Emotional & .11 & $.08(.13)$ & $-.25(-.24)$ & $.40^{* *}\left(.42^{* * *}\right)$ \\
\hline Conduct & .15 & $-.01(.05)$ & $-.08(-.07)$ & $.05(.06)$ \\
\hline Hyperactivity & .07 & $-.14(-.13)$ & $-.22(-.22)$ & $-.01(-.01)$ \\
\hline Peer problems & $.36^{* *}$ & $-.11(.01)$ & $-.16(-.15)$ & $.31(.37)$ \\
\hline Prosocial & -.27 & $.06(-.04)$ & $.10(.10)$ & $-.31(-.35)$ \\
\hline Total & .23 & $-.07(.02)$ & $-.26(-.26)$ & $.26(.29)$ \\
\hline \multicolumn{5}{|c|}{$A S D$ group $(N=42)$} \\
\hline Emotional & -.22 & $-.13(-.11)$ & $-.42^{* *}(-.37)$ & $.21(.22)$ \\
\hline Conduct & -.18 & $-.02(-.01)$ & $-.35(-.31)$ & $-.03(-.02)$ \\
\hline Hyperactivity & -.37 & $-.11(-.09)$ & $-.45^{* *}(-.37)$ & $.32(.36)$ \\
\hline Peer problems & -.13 & $-.03(-.02)$ & $-.29(-.27)$ & $.28(.29)$ \\
\hline Prosocial & .38 & $-.07(-.10)$ & $.28(.17)$ & $-.23(-.27)$ \\
\hline Total & -.35 & $-.12(-.10)$ & $-.55^{* * *}\left(-.49^{* *}\right)$ & $.30(.33)$ \\
\hline
\end{tabular}

$* * \mathrm{p}<0.01 ; * * * \mathrm{p}<0.001$

Notes: NVIQ = Non-verbal IQ; Language $=$ composite $($ mean z-score) of scores on the BPVS, CELF-CFD and TROG; SRS = Social Responsiveness Scale. 